\title{
Linking influenza epidemic onsets to covariates at different scales using a dynamical model
}

\author{
Marion Roussel Corresp., 1,2 ， Dominique Pontier ${ }^{1,2}$, Jean-Marie Cohen ${ }^{3}$, Bruno Lina ${ }^{4,5}$, David Fouchet ${ }^{1,2}$ \\ ${ }^{1}$ Laboratoire de Biométrie et Biologie Evolutive URM5558-CNRS, Université de Lyon, Université Claude Bernard Lyon 1, Villeurbanne, France \\ 2 Université Claude Bernard Lyon 1, LabEx ECOFECT Ecoevolutionary Dynamics of Infectious Diseases, Lyon, France \\ 3 OPEN ROME (Organize and Promote Epidemiological Network), Paris, France \\ 4 Laboratory of Virology, Centre National de Référence des Virus Influenzae, Hospices Civils de Lyon, Lyon, France \\ 5 Virpath, EA4610, Faculty of Medecine Lyon Est, University Claude Bernard Lyon 1, Lyon, France \\ Corresponding Author: Marion Roussel \\ Email address: marion.roussel1@gmail.com
}

Background. Evaluating the factors favoring the onset of influenza epidemics is a critical public health issue for surveillance, prevention and control. While past outbreaks provide important insights for understanding epidemic onsets, their statistical analysis is challenging since the impact of a factor can be viewed at different scales. Indeed, the same factor can explain why epidemics are more likely to begin i) during particular weeks of the year (global scale); ii) earlier in particular regions (spatial scale) or years (annual scale) than others and iii) earlier in some years than others within a region (spatiotemporal scale).

Methods. Here, we present a statistical approach based on dynamical modeling of infectious diseases to study epidemic onsets. We propose a method to disentangle the role of covariates at different scales and use a permutation procedure to assess their significance. Epidemic data gathered from 18 French regions over 6 epidemic years were provided by the Regional Influenza Surveillance Group (GROG) sentinel network.

Results. Our results failed to highlight a significant impact of mobility flows on epidemic onset dates. Absolute humidity had a significant impact, but only at the spatial scale. No link between demographic covariates and influenza epidemic onset dates could be established.

Discussion. Dynamical modelling presents an interesting basis to analyze spatiotemporal variations in the outcome of epidemic onsets and how they are related to various types of covariates. The use of these models is quite complex however, due to their mathematical complexity. Furthermore, because they attempt to integrate migration processes of the virus, such models have to be much more explicit than pure statistical approaches. We discuss the relation of this approach to survival analysis, which present significant differences but may constitute an interesting alternative for non-methodologists. 
1 Linking influenza epidemic onsets to covariates at different scales using a dynamical model

2

3 Marion Roussel ${ }^{1,2}$, Dominique Pontier ${ }^{1,2}$, Jean-Marie Cohen $^{3}$, Bruno Lina ${ }^{4,5}$, David Fouchet ${ }^{1,2}$

4

5 'Laboratoire de Biométrie et Biologie Evolutive URM5558-CNRS, Université de Lyon,

6 Université Claude Bernard Lyon 1, Villeurbanne, France.

7

8 2Université Claude Bernard Lyon 1, LabEx ECOFECT Ecoevolutionary Dynamics of Infectious

9 Diseases, F-69365 Lyon, France.

10

$11{ }^{3}$ OPEN ROME (Organize and Promote Epidemiological Network), Paris, France

12

13 4Laboratory of Virology, Centre National de Référence des Virus Influenzae, Hospices Civils de 14 Lyon, Lyon, France

15

$16{ }^{5}$ Virpath, EA4610, Faculty of Medecine Lyon Est, University Claude Bernard Lyon 1, 69372

17 Lyon Cedex08, France

19 Corresponding author: Marion Roussel

20 UMR CNRS 5558 - LBBE “Biométrie et Biologie Évolutive” UCB Lyon 1

21 Bat. Gégor Mendel 43 bd du 11 novembre 1918

2269622 VILLEURBANNE Cedex, France

23 marion.roussel1@gmail.com 


\section{ABSTRACT}

25 Background. Evaluating the factors favoring the onset of influenza epidemics is a critical public

26 health issue for disease surveillance, prevention and control. While past outbreaks provide

27 important insights for understanding epidemic onsets, their statistical analysis is challenging

28 because the impact of a factor can be viewed at different scales. Indeed, the same factor can

29 explain why epidemics are more likely to begin i) during particular weeks of the year (global

30 scale); ii) earlier in particular regions (spatial scale) or years (annual scale) than others and iii)

31 earlier in some years than others within a region (spatiotemporal scale).

32 Methods. Here, we present a statistical approach based on dynamical modeling of infectious

33 diseases to study epidemic onsets. We propose a method to disentangle the role of covariates at

34 different scales and use a permutation procedure to assess their significance. Epidemic data

35 gathered from 18 French regions over 6 epidemic years were provided by the Regional Influenza

36 Surveillance Group (GROG) sentinel network.

37 Results. Our results failed to highlight a significant impact of mobility flows on epidemic onset

38 dates. Absolute humidity had a significant impact, but only at the spatial scale. No link between

39 demographic covariates and influenza epidemic onset dates could be established.

\section{Discussion.}

41 Dynamical modelling presents an interesting basis to analyze spatiotemporal variations in the

42 outcome of epidemic onsets and how they are related to various types of covariates. The use of

43 these models is quite complex however, due to their mathematical complexity. Furthermore,

44 because they attempt to integrate migration processes of the virus, such models have to be much

45 more explicit than pure statistical approaches. We discuss the relationship of this approach to 
46 survival analysis, which present significant differences but may constitute an interesting

47 alternative for non-methodologists.

48

49 INTRODUCTION

50 Influenza is an infectious disease that causes annual epidemics around the world, inducing

51 morbidity in millions of people and a mortality of hundreds of thousands (World Health

52 Organization 2014). Influenza's ability to generate seasonal epidemics and potentially worldwide

53 pandemics makes influenza studies and surveillance a major challenge for public health

54 (Simonsen 1999). However, the mechanisms of its geographic spread and seasonality remain

55 unclear (Fuhrmann 2010; Lipsitch \& Viboud 2009). Improving our understanding of the factors

56 that trigger outbreaks is necessary for earlier detection of seasonal epidemics so that public

57 health can be better prepared and efficient preventive/control strategies can be designed.

58 From a theoretical point of view, influenza epidemic onsets are driven by two phenomena.

59 First, important external flows of infected individuals can help reach a critical number of

60 infected people. Second, local transmission conditions, such as a favorable climate and/or a high

61 density of susceptible humans, should be present.

62 From an empirical point of view, previous studies have highlighted various covariates that

63 may explain timing differences of influenza epidemics between years and areas. Human

64 movement has been suggested to impact influenza spread (Charaudeau et al. 2014; Crépey \&

65 Barthélemy 2007; Stark et al. 2012; Viboud et al. 2006). Spatial correlation of influenza

66 epidemics has been observed in major countries [USA (Viboud et al. 2006), Canada (He et al.

67 2013; Stark et al. 2012), Brazil (Alonso et al. 2007) and China (Yu et al. 2013)], but not in

68 smaller countries [Israel (Barnea et al. 2014; Huppert et al. 2012)]. Climatic covariates (Alonso 
69 et al. 2007; He et al. 2013; Shaman et al. 2010; Yu et al. 2013) and population size (Bonabeau et

70 al. 1998; Stark et al. 2012; Viboud et al. 2006) also appear to be important for epidemic onsets.

71 A certain degree of consistency in the results obtained has been observed although studies have 72 used a variety of methods and data: these are summarized in Table 1 (see Web Material 1 for a 73 discussion about the variability in data used).

74 From a methodological point of view, statistical methods applied for studying the impact 75 of covariates on epidemic onset show important differences. Most studies have used a statistical 76 approach (e.g., correlation tests (Charaudeau et al. 2014; Stark et al. 2012) or regression models 77 (Crépey \& Barthélemy 2007; He et al. 2013; Yu et al. 2013)). Only two studies (Eggo et al. 78 2010; Gog et al. 2014) employed inference based on a dynamical model to study the factors 79 affecting the geographical spread of the epidemic wave of two pandemics: Eggo et al. (2010) studied the 1918 Spanish Flu pandemic in England, Wales, and the US, and Gog et al. (2014) studied the $2009 \mathrm{H} 1 \mathrm{~N} 1$ pandemic in England. A model was used in these studies that represented the rate (probability per unit of time) at which uninfected cities become infected according to covariates (such as the proximity of infected cities, city density or humidity).

Using a model inspired by classical dynamical models of infectious disease for statistical inference is appealing because such models attempt to capture the spread mechanism of pathogens. Such models have been employed for decades to represent the spread of infectious agents (most often between individual hosts, but also between host populations (Eggo et al. 2010; Gog et al. 2014; Keeling 2002)). The second advantage is that, because the probability of entering into the epidemic state varies from week to week, epidemic onset dates can be linked to weekly variations of covariates. The use of dynamical modelling hence allows a deeper analysis

91 of epidemic onsets than purely statistical models that try to establish a correlation between 
92 epidemic onset dates and the average value of covariates across the winter period (Shaman et al. 93 2010; Yu et al. 2013).

94 In the present paper, we have analyzed the impact of five covariates that could have 95 potentially affected the time difference in the onset of epidemics between eighteen regions of 96 France over six epidemic years from 2006 to 2013 (an epidemic year corresponds to the period 97 of time from October until the following April). The five covariates analyzed were temperature 98 and absolute humidity, mobility flows, population size, and proportion of children within the 99 region. Our study is based on a dataset provided by GROG (Groupes Régionaux d'Observation 100 de la Grippe) an influenza surveillance network in France. The advantage of this network is that 101 it combines clinical case definitions with identification of the virus. This is an important 102 validation process because influenza can be clinically confounded with other co-circulating 103 respiratory viruses.

104 Our analysis has the same modeling basis as (Eggo et al. 2010; Gog et al. 2014). We put 105 particular emphasis on the idea that the impact of a factor can be viewed at different scales that 106 should be disentangled. For the studied covariates, we used permutation tests that overcome the 107 problem of non-adjustment of the dynamic epidemic models (because not all factors that affect 108 epidemic onset variability can be modeled). Indeed, by shuffling the observed values of 109 covariates, we generate random (permuted) covariates that have no biological relation to the 110 response variable (because they are random). Basically, if the observed value of a covariate 111 performs significantly better than its permuted counterparts, this means that it is correlated to the 112 response variable (even if the underlying model used in the analysis is not fully adjusted to the 113 data) 


\section{METHODS}

116 Data

117 In this analysis, the considered spatial scale is the region. The main reason for this is that the

118 GROG network, from which the data originates, provides influenza prevalence estimates at the 119 regional scale - so it was not possible to consider a lower scale here.

120

121 Epidemiological data. Epidemiological data comes from the GROG network, a French

122 surveillance network made up of voluntary General Practitioners (GPs) and pediatricians.

123 Sentinels record acute respiratory infections (ARI) weekly and randomly send nasal samples for

124 antigenic confirmation (or rejection) of influenza infection (see Web Material 2 for more detail).

125 Influenza incidence of clinical cases is then estimated as:

$$
I_{\text {influenza }}(t)=I_{A R I}(t) \times T_{+}(t)
$$

127 where $I_{A R I}(t)$ is the incidence of ARI cases and $T_{+}$is the proportion of influenza-positive samples 128 among ARI individuals. Details about the calculation of $I_{A R I}(t)$ and $T_{+}$are given in Web Material 1292.

130 Epidemiological data are available from the epidemic years of 2006-2013 for all regions of 131 metropolitan France (Web Figure 1) except Languedoc-Roussillon, Franche-Comté and

132 Limousin, where data were too scarce. Since we focus on seasonal epidemics, the 2009-2010

133 pandemic year was excluded.

134 For each year and region, we followed the GROG network procedure to define the 135 epidemic onset:

136 1. Several similar influenza viruses (AH1N1, AH3N2 and B are considered different), more 137 than what could be expected from the sporadic circulation of the virus that is observed at 
the beginning of the surveillance period, are detected or isolated in different areas of the same region;

2. At least two indicators (ARI reported by GPs + one of the 5 indicators: ARI reported by pediatricians, sick leave prescribed by GPs, GPs or emergency activity and drug distribution) increase by more than $20 \%$ compared to the average of October (of the season considered), without explanation by another phenomenon (i.e., no other local epidemic or outbreak due to other known cause);

3. A week is considered to be within an epidemic only if the previous or following week satisfies conditions 1 and 2. The epidemic onset date is defined as the first week that i) satisfies 1 and 2 and ii) is followed by a week satisfying 1 and 2 .

150 All swab results and forms were anonymized by the laboratories before they were sent to the

151 GROG network coordination, and only identified by a number given by each laboratory for

152 virological tests. In accordance with the French applicable law, clearance by an Ethics

153 Committee is not required in France for the retrospective analysis of anonymized data collected 154 within routine influenza surveillance schemes.

Mobility data. Flows of people generate contacts (including infectious ones) between populations 157 from different regions. They can therefore promote influenza spread between connected regions 158 and represent an important risk factor for regional epidemic onsets. The National Institute of Statistics and Economic Studies (INSEE) provided mobility data 160 in France. Place of residence and workplace are reported for employed individuals, while 
161 residence and school location are reported for students. We defined mobility flows as being

162 journeys between home and work or school (Figure 1). Note that these data are not representative

163 of all possible journeys (e.g., vacations, weekends). Flows were only measured between regions

164 and not at the lower scale (so, for example, travels from city 1 of region A to city 2 of region B

165 and travels from city 3 of region A to city 4 of region B are considered to be equivalent in our

166 analysis).

167

168 Demographic data. Favorable demographic characteristics of regions can also influence the

169 spread of influenza and, hence, epidemic onset. We considered two demographic metrics

170 (evaluated using INSEE data). The first metric is (the logarithm of the) population size, i.e., the

171 number of individuals living in a given region, because contacts between individuals can be

172 stronger in more populated regions, increasing the spread of the virus. We preferred considering

173 population size instead of population density, as populations are not homogeneously distributed

174 within regions (population density can be low due to large unpopulated areas despite cities

175 aggregating many individuals). The second metric is the proportion of children from 0 to 19

176 years old, this age-class being the most affected by influenza and often suspected to be a major

177 source of influenza transmission (Wallinga et al. 2006; White et al. 2014).

178

179 Climatic data. Climatic data were provided by Météo-France (the French national meteorological

180 service). We selected 125 meteorological stations (Web Figure 2) to estimate climatic covariates

181 that globally describe the climate of each region. We focused on temperature and absolute

182 humidity as climatic covariates. Even if they are correlated, they are both relevant as they might

183 impact influenza epidemics (Barreca \& Shimshack 2012; Roussel et al. 2016; van Noort et al. 
184 2012). Daily measures were averaged over the week and over the stations of a region to provide 185 weekly variable metrics in all regions.

186

187 Variability of data and covariates. Onsets of epidemics show variability at different scales 188 (Figures 2 and 3). At the global scale, epidemic onsets are more likely to occur during some 189 weeks than others, whatever region or epidemic year is considered. At the annual scale, the 190 average starting date (over regions) of epidemics varies between years. At the spatial scale, 191 epidemics can start on average (over years) earlier in some regions than in others. Without 192 additional sources of variability, we should expect to observe that some regions enter into an 193 epidemic earlier in some regions every year and earlier during some years in every region than 194 during others. In fact this is not the case, because local (a given year in a given region) specific 195 winter conditions may change the timing of epidemics. This latter scale is termed 196 spatiotemporal, because statistically it refers to an interactive effect of time and space on 197 epidemic onset dates.

To determine the scales at which epidemic onset dates and the different covariates

199 exhibit a relevant amount of variability, we performed a preliminary analysis. Let us first consider the epidemic onset date variable. We used a linear mixed model with epidemic year and region as random effects. The distribution of the random effects are considered to be Gaussian,

202 standard deviations being denoted $\sigma_{Y}$ and $\sigma_{R}$, respectively. This linear mixed model was

203 performed with the R software using the 'lme4' package, using the following command line: $\operatorname{lmer}($ EpidOnset $\sim(1 \mid$ Region $)+(1 \mid$ Year $)$, data $=$ FluOnsetData $)$ where FluOnsetData is the analyzed data set. Here the epidemic onset date was taken as a response variable (variable EpidOnset of the data set). Region and Year are the variables of the 
207 data set providing, for each observed epidemic, the associated Region and Year indexes

208 (considered as qualitative variables), respectively.

209 A similar analysis was performed using demographic variables as variable responses, using

210 the following command lines:

$$
\begin{aligned}
& \operatorname{lmer}(\text { PopSize } \sim(1 \mid \text { Region })+(1 \mid \text { Year }), \text { data }=\text { FluOnsetData }) \\
& \operatorname{lmer}(\text { PropChild } \sim(1 \mid \text { Region })+(1 \mid \text { Year }), \text { data }=\text { FluOnsetData })
\end{aligned}
$$

213 where PopSize and PropChild stand for the population size and proportion of children variables, 214 respectively.

215 For climatic covariates, weekly data are available, so we added the week variable as a

216 random effect in the linear model (the distribution of this random effect being also considered to

217 be Gaussian, with a standard deviation denoted $\widehat{\sigma_{W}}$ ), using the following line commands:

$$
\begin{aligned}
& \operatorname{lmer}(\text { Temp } \sim(1 \mid \text { Region })+(1 \mid \text { Year })+(1 \mid \text { Week }), \text { data }=\text { FluOnsetData }) \\
& \operatorname{lmer}(\text { Humid } \sim(1 \mid \text { Region })+(1 \mid \text { Year })+(1 \mid \text { Week }), \text { data }=\text { FluOnsetData })
\end{aligned}
$$

where Temp and Humid are the Temperature and humidity variables in the data set and Week is the week index associated to each measure of these two climatic variables. model outcomes, we used the 'summary' function, which provides estimations for the residual

224 variance (denoted $\hat{\sigma})$ and of the variance of random effects $\left(\hat{\sigma_{Y}}, \hat{\sigma_{R}}\right.$ and $\widehat{\sigma_{W}}$ for climatic variables)

225 for each of the five models performed.

For each of the five response variables considered, estimates of $\sigma_{Y}$ and $\sigma_{R}$ (and of $\sigma_{W}$ for

227 climatic variables) provide a good descriptive tool to account for the magnitude of associated 228 systematic variations at the different levels (systematic regional variations: $\widehat{\sigma_{R}}$, systematic inter-

229 annual variations: $\widehat{\sigma_{Y}}$ and, for climatic variables, systematic variations between week: $\widehat{\sigma_{W}}$ ). Since 
230 we do not have replicates, for each of the five linear mixed models, residual variations of the

231 model are confounded with the interaction between years and regions. For these reasons, $\hat{\sigma}$

232 quantifies the spatiotemporal standard deviation (i.e., how a given region/year deviates from

233 what could be expected from the systematic effect of regions and years) of the associated

234 variable.

235 The results of this preliminary analysis are summarized in Table 2. As epidemic onset

236 dates vary at all scales, we can potentially relate their variation to covariates at all scales.

237 Similarly, climatic covariates show important variation at all scales. Thus climatic covariates can

238 be potentially linked to epidemic onset dates at all scales.

239 Demographic covariates can vary between regions but, in our data set, change very little

240 between years. Hence trying to explain annual or spatiotemporal variation in epidemic onset with

241 demographic covariates would be pointless in our case.

242 Mobility flows are not presented in Table 2. In practice, they are assumed to be constant in

243 time. However, because we are interested in the mobility flows leading to virus exchange

244 between regions, which depend on local influenza prevalences, the associated variable will vary

245 at all scales and can be used to explain spatiotemporal variation in epidemic onsets. Therefore,

246 we will try to determine whether flows leading to virus exchanges explain regional timing of an

247 epidemic.

248 It is important to note that this preliminary analysis is completely independent of the main

249 analysis that will be presented in the next section. The use of random terms (region, year and

250 potentially, week) was important in this preliminary analysis because the objective was to

251 quantify the variability of each variable at each scale. In the main analysis, random terms will not 
252 be used because i) they were not mandatory and ii) they would render the model inference much

253 more complex.

254

255 Statistical methods

256 To analyze the link between epidemic onset dates and covariates, we used an approach based on

257 statistical inference on a dynamical stochastic epidemic model. Due to the relatively small size of

258 our data set, we reduced the number of parameters of the models as much as possible and

259 avoided random (week, epidemic year or region) factors.

260

261 The dynamical model. The dynamical model is a stochastic version of the Levin model adapted

262 to the spread of infectious diseases within a metapopulation (Keeling 2002) defined by the fact

263 that, during a small time interval $[t, t+d t]$, the probability (for a non-infected region) of entering

264 into the epidemic state for region $R$ during week $W$ of (the epidemic) year $Y$ is $\lambda(R, Y, W) d t$, where

$265 \lambda(R, Y, W)$ is the rate at which a region enters into the epidemic state (the epidemic onset rate).

266 The epidemic onset rate is modelled as the product of two terms:

267

$$
\lambda(R, Y, W)=\beta(R, Y, W) \times \phi(R, Y, W)^{\alpha}
$$

268 where $\phi(R, Y, W)$ is (any quantity that is proportional to) the flow of virus entry within region $R$

269 during week $W$ of year $Y$ and $\beta$ is a proportionality term that can depend on $R, Y$ and $W$. The

270 exponent $\alpha$ stands for the fact that the flow of virus entry might not affect the rate of epidemic

271 onset in a linear fashion. For example, epidemic triggering could require the simultaneous

272 presence of a sufficient number of infected individuals. In that case we would expect $\alpha$ to be

273 greater than one because $x$ infected individuals during $n$ subsequent weeks are less likely to

274 trigger an epidemic than $n x$ infected individuals during the same week. 
276 Mobility flows. Flows of virus entry are, to a large extent, related to flows of people between

277 regions (i.e., mobility flows). Migration of the virus from region A to region B can be related to

278 flows of people in both directions: individuals living in region A that contaminate individuals

279 from region B during their travels and/or individuals from region B that acquire the infection

280 during their travels in region A. To keep things simple, it is reasonable to assume that the

281 probability that flows from region A will lead to an epidemic in region B with a rate that depends

282 on i) the number of people flowing between A and B and ii) the proportion of people from A that

283 are carrying the virus. Because symptomatic influenza alters the behavior of infected individuals

284 (in particular their movement pattern), virus exchanges between regions are probably mostly

285 ensured by asymptomatic individuals, but it is reasonable to assume that the number of

286 asymptomatic individuals is proportional to the number of symptomatic (estimated by the GROG

287 network).

288

As a result, the function $\phi$ is modelled as follows:

$$
\phi(R, Y, W)=\sum_{i=1, i \neq R}^{N}\left(\delta_{R i}+\delta_{i R}\right) \times \frac{I_{i}(W)}{S_{i}}+c \sum_{i=1, i \neq R}^{N} \frac{I_{i}(W)}{S_{i}}
$$

290

291 where $\delta_{R i}$ and $\delta_{i R}$ correspond, respectively, to mobility flows from region $R$ to region $i$ and from 292 region $i$ to region $R$ (in number of people). $S_{i}$ represents the population size of region $i$ and $I_{i}(W)$ 293 its incidence at week $W$ (thus I/S is an estimate of the proportion of infected people). The term

$294 \sum_{i=1, i \neq R}^{N} \frac{I_{i}(W)}{S_{i}}$ is the sum of influenza prevalence over all regions except $R$. We added this term 295 because capturing the actual rate of virus exchange between two regions is complicated: the first 296 term may be inaccurate and additional virus exchanges may originate from flows other than 
297 those modelled in this term. However, because we have no way of knowing where these

298 exchanges come from, we did not make any distinction between regions (other than R) in this

299 term. This is a classical assumption in epidemic metapopulation models, the first term

300 corresponding to local transmission and the second to global transmission. $c$ is a positive

301 constant parameter that quantifies the relative weight of local and global transmission. If the

302 mobility flows we measured accurately capture the rates of virus exchanges between regions of

303 France, then $c$ should be small.

304

305 Climatic covariates. Let us consider a climatic covariate $X$ (temperature or absolute humidity)

306 that takes the value $X_{R, Y, W}$ in region $R$, in year $Y$ and week $W$. To disentangle the four scales, we

307 decompose $X$ into the sum of its mean value $\left(X_{\text {mean }}\right)$ and four sub-covariates: $X W, X R, X Y$ and

308 Xres:

309

$$
X_{R, Y, W}=X_{\text {mean }}+X W_{W}+X R_{R}+X Y_{Y, W}+\operatorname{Xres}_{R, Y, W}
$$

310 where the $X$ will be replaced by any of the two climatic covariates $(X=T$ for temperature and $311 X=H$ for humidity).

312 The mathematical definition of the four sub-covariates and their biological interpretation

313 are the following (please note that for all weekly averages, the average is calculated over the

314 period starting in October of one year and ending in March of the following year). $X W_{W}$ denotes the average value of $X_{R, Y, W}-X_{\text {mean }}$ over the different regions and the different

316 years. $X W$ represents the overall (over all regions and years) global variation value of $X$. For 317 example, if $T W=4$, this means that the average temperature during week $W$ is four (Celsius) 318 degrees above the average value of the temperature over the epidemic period. Week $W$ is 319 globally four degrees warmer than the average. Because $X W$ measures the variations in the 
320 average temperature over weeks, it may explain variations in epidemic onset dates at the global

321 scale (i.e., why epidemic onsets are more likely to occur some weeks than others). The objective

322 here is to evaluate whether the average timing of influenza in the epidemic year is linked to

323 average climatic conditions.

$324 \quad X R_{R}$ denotes the average value of $X_{R, Y, W}-X_{\text {mean }}$ over the different weeks of the epidemic

325 period and all years. $X R$ represents regional systematic differences. For example, $T R=2$ means

326 that the average (over all weeks and years) temperature in region $R$ is two degrees above the

327 average temperature over all weeks, years and regions. Region $R$ is globally two degrees warmer

328 than the average. The sub-covariate $X R$ can explain epidemic onset variation at the spatial scale.

329 The objective is to evaluate whether the time differences of influenza epidemic onsets between

330 regions can be explained by different average climatic conditions between the regions.

$331 X Y_{Y, W}$ denotes the average value of $X_{R, Y, W}-\left(X_{\text {mean }}+X W_{W}\right)$ over the different regions. $X Y$

332 stands for annual global differences. For example, $X Y_{Y, W}=-5$ means that during year $Y$, the

333 average temperature values that have been observed during week $W$ over all regions is five

334 degrees below the average values of temperature that have been observed over all regions and

335 years during the same week $W$. If during year $Y$ all values of $X Y$ are positive (during all weeks),

336 this means that the winter of epidemic year $Y$ is globally warmer than the average. If $X Y$ is

337 negative during several subsequent weeks, it may reveal a cold snap in that period. Thus $X Y$ not

338 only summarizes the average value of the covariate during the winter but also whether there have

339 been some periods in the winter when the covariate was high and/or low (early epidemic onsets

340 may simply arise from specific climatic conditions within limited time windows). It can explain

341 variations of epidemic onset dates at the annual scale (i.e., why epidemics start on average earlier

342 some years than others). 
Finally, $X \operatorname{rres}_{R, Y, W}=X_{R, Y, W}-\left(\left(X_{\text {mean }}+X W_{W}+X R_{R}+X Y_{Y, W}\right)\right.$ represents spatiotemporal weekly

344 residual variations. For example, $\operatorname{Tres}_{R, Y, W}=-3$ means that, considering the average temperature

345 values that where observed during week $W$ of year $Y$ in all regions on one hand, and the global

346 characteristic of region $R$ compared to other regions on the other, the observed value of

347 temperature in region $R$, week $W$ and year $Y$ is three degrees below what could have been

348 expected. So Xres informs us about the local characteristics of a particular winter in each region

349 and can be linked to variations in epidemic onset dates at the spatiotemporal scale.

350

351 The complete model for $\beta$. The proportionality term $\beta$ can be different between regions, years

352 and weeks because, considering a given flow of virus entry, local conditions within the region

353 can, during a particular week, increase or decrease the risk of entering into an epidemic state. So

$354 \beta$ can depend on several covariates, including demographic and climatic. The complete model

355 (that integrates all the measured covariates) is defined by:

356

$\log (\beta(R, Y, W))$

$=a_{0}+a_{S} \times \log \left(S_{R}\right)+a_{C} \times C_{R}+a_{T W} \times T W_{W}+a_{T R} \times T R_{R}+a_{T Y} \times T Y_{Y, W}+a_{T r e s}$

$\times \operatorname{Tres}_{R, Y, W}+a_{H W} \times H W_{W}+a_{H R} \times H R_{R}+a_{H Y} \times H Y_{Y, W}+a_{\text {Hres }} \times$ Hres $_{R, Y, W}$

where $S$ and $C$ represent respectively, the region population size and proportion of children. Note

360 that since demographic covariates show little inter-annual variation, they are only likely to

361 explain spatial variability in epidemic onsets. For that reason, we considered the average value of

362 these covariates over all years in each region as model covariates. Parameters $a$ are model

363 constant coefficients that quantify the link between each covariate and $\beta$. To allow a direct 
364 comparison between all the coefficients a, the four covariates $(S, C, T$ and $H)$ have been centered

365 and standardized before the analysis. Coefficient $a_{0}$ is the intercept of the model.

366

367 Model likelihood

368 Model parameters were estimated using a maximum likelihood procedure. The link between

369 epidemic onset dates and model covariates was tested using the likelihood-ratio test (LRT)

370 statistic. The chi-square approximation of the LRT was not used here because it requires both

371 large sample size and assumes that data can be considered as a plausible outcome of the model

372 (i.e., model adjustment). In our case, model adjustment requires all potential sources of weekly,

373 inter-annual and inter-regional variations to be incorporated in the model. Because this was not

374 the case - we did not include random terms in our model - we preferred not to rely on this

375 approximation. Instead, permutation tests were used (see below).

376 For an epidemic year $Y$, the probability of a region $R$ to enter into an epidemic state in a

377 particular week $W$ is given by the probability that the region did not enter into an epidemic state

378 before week $W-1: e^{-\sum_{i=0}^{W-1} \lambda(R, Y, i)}$ and the probability that the epidemic occurs during the week that

379 started at $W: 1-e^{-\sum_{i=0}^{W-1} \lambda(R, Y, i)}$. That is why the likelihood $(L)$ of a region $R$ and an epidemic year

$380 Y$ is defined as:

381

$$
L=e^{-\sum_{i=0}^{W-1} \lambda(R, Y, i)} \cdot\left(1-e^{-\lambda(R, Y, W)}\right)
$$

383 epidemic year, given by:

384

$$
L_{g}=\prod_{R, Y} e^{-\sum_{i=0}^{W-1} \lambda(R, Y, i)} \cdot\left(1-e^{-\lambda(R, Y, W)}\right)
$$


Model parameters were inferred using maximum likelihood estimation. Models and

386

387

388

389

390

391

392

393

394

395

396

397

398

399

400

401

402 Permutation tests

403

404

405

406

Permutation tests

permutation tests were implemented in Matlab.

It should be noted that, due to an insufficient covering during some weeks in some regions, influenza incidence could not be estimated for these points. Because the statistical procedure requires incidence values to calculate the terms associated with mobility flows, we replaced missing incidence values by zeros in the program.

data points in the analysis is feasible (under its current form, the Matlab code integrates this possibility). However, including them altered the results of the analysis in a way that we think is counterproductive (see Web Material 3 for more details), so we preferred to exclude them from the analysis. From a biological point of view, this choice is reasonable because it is likely that these regions/years present specific characteristics (e.g., an important proportion of immune individuals) meaning that, despite an important flow of virus entry, they could not enter into the epidemic state. This case scenario was not integrated in the model, which assumes that, provided a sufficient flow of virus entry, any region could enter into the epidemic state during any season.

Permutation tests are based on the idea that randomly shuffling the values of a covariate $F$ looks at the distribution of the possible linkages that could have been found between $\lambda$ and the covariate $F$ given data. Hence, replicates of random shuffling of the values of $F$ can be used to estimate the distribution of the LRT under $\mathrm{H}_{0}$ 'no impact of the covariate' (Lebreton et al. 2012). 
407 An interesting property of covariate (rather than data) shuffling is that other covariates can

408 remain unshuffled and keep their ability to reduce residual variance.

409 Because several covariates vary according to only one index $(W, R$ or $Y)$, we used block

410 permutations - covariates were shuffled according to some indexes but not others - to keep the

411 error structure of covariates. For example, population size $(S)$ varies only between regions.

412 Hence, the associated permutation test shuffles the values of $S$ between regions but keeps it

413 constant between weeks and years. According to their scale of variation, all covariates were

414 tested according to a specific set of indexes (Table 3).

415 The four following steps can summarize the principle of permutation tests:

416 Step 1: shuffle randomly a covariate. Potentially, variables have three indexes of

417 variations: weeks $(W)$, year $(Y)$ and region $(R)$. Let us call $P$ a random permutation of the triplet

$418(W, Y, R)$ (the different types of permutation that can be used will be detailed below). Let us call $X$

419 the covariate that has to be permuted. The original (non-permuted) covariate is $X_{W, Y, R}$. The

420 permuted covariate is called $Z$ and is defined by $Z_{W, Y, R}=X_{P(W, Y, R)}$.

421 Step 2: determine the test statistics associated with each permutation. We used the

422 likelihood ratio test (LRT), defined as $-2 \times \log \left(\frac{L_{Z}}{L_{0}}\right)$, where $L_{Z}$ and $L_{0}$ respectively represent the

423 likelihoods of models with and without covariate $Z$. Note that, for mobility flows, the model

424 without this term is not used (the associated coefficient always equals one). In that case, the LRT

425 statistic used is replaced by the deviance (defined by $-2 \log \left(L_{Z}\right)$ ) statistic, other steps being

426 unchanged.

427 Step 3: determine the distribution of the LRT statistic under the null hypothesis H0:

428 "epidemic onsets are independent of covariate $X$ '. Since permutations generate random

429 covariates that have no biological reason to be associated with epidemic onsets, each permutation 
430 represents a random realization of the LRT statistic under H0. For each covariate $X, 1,000$

431 permutations were generated and Step 1 and Step 2 led to 1,000 independent values of the LRT

432 under H0. From that we could derive an estimate of the distribution of the LRT under H0.

433 Step 4: determine a threshold for the LRT under H0. The threshold was simply taken as the

$43495 \%$ quantile of the distribution of permuted LRTs. Comparing the observed value of the LRT

435 with this threshold provides a test criterion for rejecting, or not, $\mathrm{H} 0$.

436 Alternatively, we can estimate a $p$ value for each test, defined as $p=(x+1) /(N+1)$, where $x$

437 is the number of permuted values of the LRT above that observed and $N=1,000$ is the number of

438 permutations. $\mathrm{H} 0$ is then rejected as soon as $p<0.05$ but is otherwise accepted.

439

Based on the level at which we want to establish correlates between epidemic onset dates

441 and covariates, different tests have to be performed. If we want to test a covariate that explains

442 epidemic onset variations at the spatial level, only region indexes will be shuffled. In practice, let

443 us call $P_{R}$ a permutation of region indexes, then a permutation shuffling only regions indexes

444 will take the form of $P(W, Y, R)=\left(W, Y, P_{R}(R)\right)$. Shuffling only region indexes means that measures

445 are repeatedly the same each year and each week within a region.

446 Similarly, shuffling only year indexes will test covariates explaining annual variations in

447 epidemic onsets. Let us call $P_{Y}$ a permutation of years, the permutation taking the form:

$448 P(W, Y, R)=\left(W, P_{Y}(Y), R\right)$. In the same way, shuffling week indexes will test covariates explaining

449 global variations (why epidemic onset does not happen randomly within the studied period). By

450 calling $P_{w}$ a permutation of the week, the permutation will take the form $P(W, Y, R)=\left(P_{W}(W), Y, R\right)$.

451 For climatic covariates explaining spatiotemporal variations in epidemic onsets, we chose

452 to independently shuffle region and year indexes. In practice, the permutation will take the form 
453 of $P(W, Y, R)=\left(W, P_{Y}(Y), P_{R}(R)\right)$. Shuffling region and year indexes independently rather than

454 simultaneously has the advantage of keeping the general intra-annual and intra-regional

455 structures in covariates.

456 Finally, for the mobility covariate permutations, we first shuffled regions (in the $\delta$ matrix,

457 similar permutations were used for lines and columns of the matrix) and then recalculated the

458 (permuted) flow of people between all pairs of regions (coefficients $\delta$ ). Then the flow of infected

459 people was calculated by multiplying these coefficients by the non-permuted regional

460 prevalence, leading (for all regions, years and weeks) to a new value for the first term of $\phi$ (i.e.,

$\left.461 \sum_{i=1, i \neq R}^{N}\left(\delta_{R i}+\delta_{i R}\right) \times \frac{I_{i}(W)}{S_{i}}\right)$. The advantage of this choice is that it tells us how re-associating

462 regions randomly explains the observed synchrony between connected regions. Permuting the

463 region indexes allows us to keep the structure of the global connection network of the country

464 (e.g., the fact that some regions are more connected to other regions than others). In summary,

465 the connection network between the regions remains the same in permuted data but their link to

466 epidemic onset probabilities is broken.

467

468

One important question when testing the link between a response variable and covariates is

469 the set of correction covariates that should be introduced. One way to deal with this question is to

470 use the complete model and remove the covariate we want to test. This solution is interesting

471 because, if the test turns out to be significant, then the link between the response variable and the

472 covariate that is observed cannot be explained by any confounding effect of the other covariates.

473 Considering our relatively low sample size, this is not the solution we retained here because it is

474 conservative, especially when covariates are correlated (which is, e.g., the case for temperature

475 and humidity). Instead, for each covariate, the link was tested without correcting by all the 
476 covariates that have the same scale of variation. The other covariates were kept because they can

477 capture some of the epidemic onset date variability.

478 The case of mobility flow is singular because this variable is included as a correction

479 covariate in all models and it is not associated with any model parameter. Permutation tests were

480 also performed on this covariate (see above). We performed two different tests. In the first

481 (termed 'corrected') we kept all other covariates as correction terms (so we use the complete

482 model). In the second (termed 'uncorrected'), we removed all the other (demographic and

483 climatic) covariates.

484

485

\section{RESULTS}

486 The main model parameters (that quantify the impact of the studied covariates) are given in 487 Table 4, together with the associated $p$ value of the corresponding test. A table summarizing all 488 the model parameters inferred from all the different models used can be found in Web Table 1.

489 Covariates are considered to be significantly linked to epidemic onset dates as soon as the 490 associated $p$ value falls below 5\%. Figures showing the distribution of the LRT statistic are given 491 in Web Figures 3-6.

492 Absolute humidity was found to be significantly linked to epidemic onset dates at the 493 spatial scale $(\mathrm{p}=0.029)$, but not at the other scales. The associated coefficient was negative ($494 \quad 0.4763)$

495 Mobility flows were not found to be significantly linked to epidemic onset dates $(p=0.57$ 496 with the corrected model, $\mathrm{p}=0.73$ with the uncorrected model). In the corrected model, the 497 coefficient associated with global incidence was very high, even when we considered that the 498 local transmission term was multiplied by mobility flows (whose average is around 14,400). 
499 Such an important weight of the global incidence is not found in the uncorrected model were we

500 removed all covariates (although the test of mobility flows remained not significant, see Web

501 Table 1). This suggests that the combination of covariates used in the complete model best

502 explains spatiotemporal variation than those explained by mobility flows.

503 Population size and proportion of children were not significantly linked to epidemic onset

504 dates at the spatial scale.

505

506

\section{DISCUSSION}

507 We have presented an approach inspired by the dynamical modeling presented in (Eggo et al.

508 2010; Gog et al. 2014) to test and quantify the link between several covariates and the onset date

509 of epidemic influenza in France. The objective was both to provide new insights in influenza

510 epidemic knowledge and, more generally, to discuss the issue of the multiple scales by which the

511 link can be viewed and propose permutation tests associated with each level of variation.

512

513 Impact of mobility flows and demographic covariates

514 Our results did not reveal an impact of mobility flows on epidemic onset dates. This is quite

515 surprising because mobility flows of infected individuals between regions can help the

516 accumulation of a critical number of infected people leading to the influenza outbreak. Previous

517 studies showed a correlation between daily work commutes and global influenza spread as well

518 as regional epidemic peaks in France (Charaudeau et al. 2014; Crépey \& Barthélemy 2007) and

519 also in USA (Crépey \& Barthélemy 2007; Stark et al. 2012; Viboud et al. 2006). The fact that we

520 did not observe this link in our study may be due to inaccurate estimates of these flows. Simply

521 considering flows of workers and students (and not those linked to holidays and week-ends) 
522 could be too simplistic. The spatial scale at which we worked (the region) could also be too

523 narrow to view the spatial spread of the virus.

524 Children are also central to the spread of a disease like influenza. They are the most

525 aggregated age-class of the human population and have a relatively naïve immune system (in

526 terms of immune memory). Consistently, several studies (Peters et al. 2014; Schanzer et al. 2011;

527 Stockmann et al. 2013; Timpka et al. 2012) have reported earlier epidemics in school-age

528 children than in other age groups. Furthermore, in England (Pebody et al. 2015) and in Florida

529 (Tran et al. 2014), vaccination of school age children has been shown to reduce influenza

530 incidence in all age-classes as well reducing excess respiratory mortality, stressing the role of

531 children in influenza transmission. We have not found any statistical association between

532 demographic covariates and epidemic onset dates.

533

534 Climatic covariates: a typical example of a multi-scale issue

535 Climate is also an important factor for virus spread. It affects virus survival outside the host

536 (Lofgren et al. 2007; Lowen et al. 2007), host susceptibility to the infection (Eccles 2002) and

537 human behavior (Lofgren et al. 2007). Studying its impact on influenza epidemic onsets is hence

538 relevant, but as it can be viewed at different scales, its analysis is more complex.

539 In eco-epidemiology (and in ecology in general), it is more and more common to deal with

540 data acquired at multiple scales (spatial, temporal, populational, individual, etc.). Such data

541 present a methodological challenge because covariates may explain the variability of data at

542 different scales. In our example, epidemic onsets showed four levels of variability. At the highest

543 level (global), climate may explain why influenza epidemics occur more frequently in some

544 weeks than in others. At the spatial scale (respectively, annual), they may explain why influenza 
545 epidemics start earlier on average in some regions (respectively, years) than in others. At the

546 lowest scale (spatiotemporal), local climatic conditions could explain why an epidemic occurs

547 earlier or later in a given year in a given region.

548 In general, larger scales are associated with the more confounding effects. Systematic

549 changes in climate between regions also come with systematic changes in other covariates (such

550 as demography, economy, etc). Similarly, systematic shifts in climate between years come with

551 shifts in, e.g., antigenic characteristics of influenza strains, human society characteristics (that

552 evolve in parallel with climate changes). All these covariates can introduce statistical confusion

553 in the interpretation of model inference.

554 The smallest scale, where we try to link deviations in epidemic onset with deviations in

555 climate (after accounting for systematic variations in yearly and regional average climate), would

556 in our case be the ideal statistical scale. However, it also comes with more noise in variable

557 estimates, which is reduced at the upper scales (which are averages).

558 The only scale at which the impact of climate was found to be significant here was the

559 spatial scale for humidity. This means that, in region with dry climates, epidemics of influenza

560 tend to start earlier. However, the $p$ value associated with this covariate was close to $5 \%$ and one

561 could wonder whether the link could be artificial considering the number of tests we performed

562 in our analysis. In any case, it is interesting to note that, for all climatic covariates whose

563 coefficient was not close to zero, all values were negative, which is consistent with the idea that

564 dry and cold climates promote the spread of influenza.

565

566 Methodological issues 
567 Dynamical modeling offers a natural basis for understanding the spread of infectious diseases.

568 Paired with statistical tools, they have been used with success to analyze the spread of infectious

569 agents within non-spatialized (Chowell et al. 2004; Gibson et al. 2004) as well as spatialized

570 (Fang et al. 2016; Gibson 1997; Merler et al. 2015) host populations. However, because they are

571 based on the modelling of the mechanisms underlying the spread of agents, such approaches

572 raise important methodological issues.

573 Linking the probability of epidemic onset to weekly shifts in climatic covariates is

574 appealing but requires accurate onset date estimates. Because the climate can change rapidly

575 during the winter in France, a lag of a few weeks between the real and observed onset dates

576 weakens the strength of its link with climatic covariates. The major difficulty with observational

577 estimates of epidemic onset dates is that they are based on a clinical criterion (atypical increases

578 in influenza infection). If this choice is legitimate from a management point of view, it does not

579 necessarily translate the real epidemiologic point when all conditions are gathered to ensure the

580 massive spread of the disease and a time lag may exist between this 'break point' and the

581 estimated point.

582 Another important point regarding epidemiological models is that, at least in our case, they

583 cannot perfectly describe the variability of the response variable. This would require capturing

584 all the variations of the probability of epidemic onset between weeks, years and regions. Within

585 a simple dynamical model, it is unfortunately not possible to account for all the complexity of

586 the transmission process. Vacations were not included in the analysis. Integrating them would

587 have been complex because, in France, regional vacations are not synchronized. Vacations affect

588 the spread of a virus like influenza in a complex way (Cauchemez et al. 2008). Schools are 
589 closed and travel patterns are changed, and travel associated with work or study is replaced by

590 tourism. Unfortunately, we had no such fine information in our data set.

$591 \quad$ Network coverage was also an important issue. Three regions could not be studied for this

592 reason and, in others, we had some points missing in our prevalence estimates. This can have

593 implications for the estimate of virus entry within regions, missing points being potentially

594 associated to unquantified flows of virus entry. However, because missing data were mainly

595 associated with poorly connected regions and/or to periods of the year when influenza

596 prevalence is low, we believe that neglecting them is not too prejudicial for the analysis.

597 It is important to remind that, for some epidemic years in some regions, no epidemic of

598 influenza was observed. For reasons detailed in Web material 3, we chose to remove these

599 regions from our analysis. This implies that our results are only relevant for understanding the

600 link between influenza epidemic onset dates and covariates for regions and epidemic years for

601 which an epidemic did occur and should not be extrapolated to explain why no epidemic

602 occurred in some circumstances.

603 Another important point to discuss in such an analysis is the geographical scale at which

604 data are measured. Due to the spatial covering of the GROG network, it was not possible to work

605 below the regional level. We are conscious that many phenomena may occur at lower scales:

606 regions are not homogeneous in terms of human density, movement patterns and climate.

607 However, because this problem is due to the basic structure of the data, there was not much we 608 could do.

609

610 For all these reasons it was important not to rely on the asymptotic assumption of the chi611 square distribution of the likelihood ratio statistic. Such an assumption is only valid when the 
612 model is able to describe the complexity of the variations of the response variable (here the

613 epidemic onset rate). Here, this would have been a very strong assumption, as we can see on

614 Web Figures 3-6 (where the 95\% rejection thresholds are quite different from what we would

615 have observed with a chi-square approximation of the likelihood ratio statistic). In such a

616 context, permutation tests appear to be a very interesting tool to overcome the issue of model

617 adjustment. Indeed, permutation tests of covariate focus on the distribution of the covariate

618 (which is simple) and not on that of the response variable (which is complex). Thus, even if the

619 underlying model is incorrect, permuted covariates have absolutely no reason to perform better

620 than those observed. They offer therefore, a robust means to test the impact of the different

621 covariates.

622 If permutation tests reduce the risk related to robustness of the analysis to depart from

623 model assumptions, they also have some drawbacks. They require a lot of computation time to

624 perform a large number of permutations, each one requiring involving the recomputation of the

625 test statistic. Also, they consider fixed observed values for all the variables, evaluating whether

626 the pattern observed in the data is likely, or not, to have arisen by chance. The underlying theory

627 of permutation tests is hence not based on the random sampling assumption (made in parametric

628 approaches), which has the advantage that the conclusions of the analysis can be generalized to

629 the entire population (Ernst 2004). So in contrast, from a theoretical point of view, permutation

630 tests only allow to draw conclusions that are relevant to the particular data set.

631 In addition, permutation tests do not resolve the important problem of statistical power.

632 The data set we analyzed here is relatively small (around a hundred points). Because our

633 approach is relatively new, it is hard to know whether such a data set is sufficient for a

634 reasonable statistical power. 
635 The lack of statistical power is probably the reason why we found so few associations in

636 our analysis. So it is important to note that our inability to detect effects is far from proving their

637 absence. We believe that our study suggests a novel means to treat epidemic onset data by

638 combining dynamical modeling with hypothesis testing based on permutation tests of the

639 covariates.

640 Testing the significance of the observed associations is already a complex task by itself, so

641 in the present paper we chose not to address the issue of evaluating confidence intervals for our

642 model parameters. In our case, such intervals would not be very insightful because we found

643 only one significant association (with a $p$ value that is close to the rejection threshold, raising the

644 question of multiple testing effects).

645 As a future direction, permutation tests provide an interesting way to evaluate equivalents

646 of confidence intervals (LaMotte \& Volaufova 1999). Such intervals are quite complex to

647 implement and are still marginal in the literature but present the advantages of permutation tests

648 that we exposed earlier.

649

650 Link with the survival analysis approach

651 Using dynamical modeling may appear rather complex to non-methodologists because of the

652 lack of existing software packages to implement such models. Handmade programs are also

653 exposed to programming mistakes. Although we carefully checked our program, such mistakes

654 could not be excluded.

655 For people who (arguably) prefer methods based on long-term existing software packages,

656 an interesting comparison can be made between our approach and (Cox regression) survival

657 analysis models. The modeling basis of both approaches are the same. The rate of epidemic onset 
658 is similar to the hazard function. Cox regression uses linear links between the logarithm of the

659 hazard function and covariates. Our link is slightly more complex, the only source of non-

660 linearity lying in the fact that we sum the local and global flows of virus entry. Here,

661 linearization of the relationship between the logarithm of the epidemic onset rate and covariate

662 could be achieved with only a few approximations.

663 However, it is important to note there is an important difference between our analysis and

664 Cox regression survival analysis that involves the way in which likelihood is calculated. Cox

665 regression uses partial likelihood. Basically, partial likelihood consists of comparing the value of

666 covariate every time an event occurs. Thus the Cox regression model finds the best linear

667 combination of covariates that maximize the probability that, considering that several events

668 could have occurred on a given date, the observed event (associated with the date) was the one

669 that occurred. So partial likelihood does not try to explain why events occurred on the precise

670 date that they did occur but why they occurred in a given order.

671 In contrast, the way we calculated likelihood here integrates this information. So for

672 example, if an epidemic onset occurred at the beginning of December in a given region during a

673 given year, our method tries to find the combination of covariates that best explains why the

674 onset did not occur earlier (for example by trying to link it to specific climatic conditions that

675 were present at the beginning of December but not in November).This is quite different from

676 what is done with the partial likelihood of the Cox regression.

677 Which way of calculating likelihood is better is still unclear due to the absence (to our

678 knowledge) of theoretical studies comparing both approaches. It is all a matter of which pieces of

679 information we want to include to infer model parameters. The Cox regression has the advantage

680 of being implemented in many classical software routines of data analysis (such as R). Thus, for 
681 researchers who are inspired by our approach to analyze epidemic onset data, adapting our model

682 (basically by linearizing the relationship between the logarithm of the epidemic onset rate and

683 covariates) to the Cox regression framework could represent an interesting compromise to

684 overcome the programming issues associated with our approach.

685

686

687

\section{ACKNOWLEDGEMENTS}

688 We acknowledge the practitioners of the sentinel network Réseau des GROG and the labs

689 involved in the surveillance. We thank Isabelle Daviaud from Open Rome who sorted the

690 epidemiological data from the sentinel network Réseau des GROG and Annick Auffray from

691 Météo-France for her kind help with meteorological data. We thank Robin Buckland for proofing

692 the English manuscript. This work was archived using the computing facilities of CC

693 LBBE/PRABI and CC IN2P3. It was performed within the framework of the LABEX ECOFECT

694 (ANR-11-LABX-0048) of the University of Lyon, within the program 'Investissements

695 d'Avenir' (ANR-11-IDEX-0007) operated by the French National Research Agency (ANR).

696

697 REFERENCES

698 Alonso WJ, Viboud C, Simonsen L, Hirano EW, Daufenbach LZ, and Miller MA. 2007.

699 Seasonality of Influenza in Brazil: A Traveling Wave from the Amazon to the

700

701

702

703

704

705

706

707

708 Subtropics. American Journal of Epidemiology 165:1434-1442.

Barnea O, Huppert A, Katriel G, and Stone L. 2014. Spatio-Temporal Synchrony of Influenza in Cities across Israel: The "Israel Is One City" Hypothesis. PLoS ONE 9:e91909.

Barreca AI, and Shimshack JP. 2012. Absolute Humidity, Temperature, and Influenza Mortality: 30 Years of County-Level Evidence from the United States. American Journal of Epidemiology 176:S114-S122.

Bonabeau E, Toubiana L, and Flahault A. 1998. The geographical spread of influenza. Proceedings of the Royal Society of London Series B: Biological Sciences 265:24212425. 
709

710

711

712

713

714

715

716

717

718

719

720

721

722

723

724

725

726

727

728

729

730

731

732

733

734

735

736

737

738

739

740

741

742

743

744

745

746

747

748

749

750

751

752

753

754

Cauchemez S, Valleron A-J, Boelle P-Y, Flahault A, and Ferguson NM. 2008. Estimating the impact of school closure on influenza transmission from Sentinel data. Nature 452:750-754. http://www.nature.com/nature/journal/v452/n7188/suppinfo/nature06732_S1.h $\underline{\mathrm{tml}}$

Charaudeau S, Pakdaman K, and Boëlle P-Y. 2014. Commuter Mobility and the Spread of Infectious Diseases: Application to Influenza in France. PLoS ONE 9:e83002.

Chowell G, Hengartner NW, Castillo-Chavez C, Fenimore PW, and Hyman JM. 2004. The basic reproductive number of Ebola and the effects of public health measures: the cases of Congo and Uganda. Journal of Theoretical Biology 229:119-126. https://doi.org/10.1016/j.jtbi.2004.03.006

Crépey P, and Barthélemy M. 2007. Detecting Robust Patterns in the Spread of Epidemics: A Case Study of Influenza in the United States and France. American Journal of Epidemiology 166:1244-1251.

Eccles R. 2002. An Explanation for the Seasonality of Acute Upper Respiratory Tract Viral Infections. Acta Oto-laryngologica 122:183-191.

Eggo RM, Cauchemez S, and Ferguson NM. 2010. Spatial dynamics of the 1918 influenza pandemic in England, Wales and the United States. Journal of The Royal Society Interface 8:233-243.

Ernst MD. 2004. Permutation Methods: A Basis for Exact Inference. Statist Sci 19:676-685. $10.1214 / 088342304000000396$

Fang L-Q, Yang Y, Jiang J-F, Yao H-W, Kargbo D, Li X-L, Jiang B-G, Kargbo B, Tong Y-G, Wang Y-W, Liu K, Kamara A, Dafae F, Kanu A, Jiang R-R, Sun Y, Sun R-X, Chen W-J, Ma M-J, Dean NE, Thomas H, Longini IM, Halloran ME, and Cao W-C. 2016. Transmission dynamics of Ebola virus disease and intervention effectiveness in Sierra Leone. Proceedings of the National Academy of Sciences 113:4488-4493. $10.1073 /$ pnas. 1518587113

Fuhrmann C. 2010. The Effects of Weather and Climate on the Seasonality of Influenza: What We Know and What We Need to Know. Geography Compass 4:718-730.

Gibson GJ. 1997. Markov Chain Monte Carlo Methods for Fitting Spatiotemporal Stochastic Models in Plant Epidemiology. Journal of the Royal Statistical Society: Series $C$ (Applied Statistics) 46:215-233. 10.1111/1467-9876.00061

Gibson GJ, Kleczkowski A, and Gilligan CA. 2004. Bayesian analysis of botanical epidemics using stochastic compartmental models. Proceedings of the National Academy of Sciences of the United States of America 101:12120-12124. 10.1073/pnas.0400829101

Gog JR, Ballesteros S, Viboud C, Simonsen L, Bjornstad ON, Shaman J, Chao DL, Khan F, and Grenfell BT. 2014. Spatial Transmission of 2009 Pandemic Influenza in the US. PLoS Computational Biology 10:e1003635.

He D, Dushoff J, Eftimie R, and Earn DJD. 2013. Patterns of spread of influenza A in Canada. Proceedings of the Royal Society of London B: Biological Sciences 280.

Huppert A, Barnea O, Katriel G, Yaari R, Roll U, and Stone L. 2012. Modeling and Statistical Analysis of the Spatio-Temporal Patterns of Seasonal Influenza in Israel. PLoS ONE 7:e45107.

Keeling MJ. 2002. Using individual-based simulations to test the Levins metapopulation paradigm. Journal of Animal Ecology 71:270-279.

Peer] reviewing PDF | (2016:11:14278:4:0:NEW 9 Feb 2018) 
755

756

757

758

759

760

761

762

763

764

765

766

767

768

769

770

771

772

773

774

775

776

777

778

779

780

781

782

783

784

785

786

787

788

789

790

791

792

793

794

795

796

797

798

799

LaMotte LR, and Volaufova J. 1999. Prediction Intervals Via Consonance Intervals. Journal of the Royal Statistical Society: Series D (The Statistician) 48:419-424. 10.1111/1467-9884.00200

Lebreton JD, Choquet R, and Gimenez 0. 2012. Simple Estimation and Test Procedures in Capture-Mark-Recapture Mixed Models. Biometrics 68:494-503. 10.1111/j.15410420.2011.01681.x

Lipsitch M, and Viboud C. 2009. Influenza seasonality: Lifting the fog. Proceedings of the National Academy of Sciences 106:3645-3646.

Lofgren E, Fefferman NH, Naumov YN, Gorski J, and Naumova EN. 2007. Influenza Seasonality: Underlying Causes and Modeling Theories. Journal of Virology 81:54295436.

Lowen AC, Mubareka S, Steel J, and Palese P. 2007. Influenza Virus Transmission Is Dependent on Relative Humidity and Temperature. PLoS Pathogens 3:e151.

Merler S, Ajelli M, Fumanelli L, Gomes MFC, Piontti APy, Rossi L, Chao DL, Longini IM, Jr., Halloran ME, and Vespignani A. 2015. Spatiotemporal spread of the 2014 outbreak of Ebola virus disease in Liberia and the effectiveness of non-pharmaceutical interventions: a computational modelling analysis. THE LANCET Infectious Diseases 15:204-211. 10.1016/S1473-3099(14)71074-6

Pebody RG, Green HK, Andrews N, Boddington NL, Zhao H, Yonova I, Ellis J, Steinberger S, Donati M, Elliot AJ, Hughes HE, Pathirannehelage S, Mullett D, Smith GE, de Lusignan S, and Zambon M. 2015. Uptake and impact of vaccinating school age children against influenza during a season with circulation of drifted influenza A and B strains, England, 2014/15. Eurosurveillance 20:1-11.

Peters TR, Snively BM, Suerken CK, Blakeney E, Vannoy L, and Poehling KA. 2014. Relative timing of influenza disease by age group. Vaccine 32:6451-6456.

Roussel M, Pontier D, Cohen J-M, Lina B, and Fouchet D. 2016. Quantifying the role of weather on seasonal influenza. BMC Public Health 16:441. 10.1186/s12889-0163114-x

Schanzer D, Vachon J, and Pelletier L. 2011. Age-specific Differences in Influenza A Epidemic Curves: Do Children Drive the Spread of Influenza Epidemics? American Journal of Epidemiology 174:109-117.

Shaman J, Pitzer VE, Viboud C, Grenfell BT, and Lipsitch M. 2010. Absolute Humidity and the Seasonal Onset of Influenza in the Continental United States. PLoS Biology 8:e1000316.

Simonsen L. 1999. The global impact of influenza on morbidity and mortality. Vaccine 17, Supplement 1:S3-S10.

Stark JH, Cummings DAT, Ermentrout B, Ostroff S, Sharma R, Stebbins S, Burke DS, and Wisniewski SR. 2012. Local Variations in Spatial Synchrony of Influenza Epidemics. PLOS ONE 7:e43528.

Stockmann C, Pavia AT, Hersh AL, Spigarelli MG, Castle B, Korgenski K, Byington CL, and Ampofo K. 2013. Age-Specific Patterns of Influenza Activity in Utah: Do Older School Age Children Drive the Epidemic? Journal of the Pediatric Infectious Diseases Society 3:163-167.

Timpka T, Eriksson O, Spreco A, Gursky EA, Strömgren M, Holm E, Ekberg J, Dahlström O, Valter L, and Eriksson H. 2012. Age as a Determinant for Dissemination of Seasonal 
800

801

802

803

804

805

806

807

808

809

810

811

812

813

814

815

816

817

818

819

820

821

822

823

824

825

826 and Pandemic Influenza: An Open Cohort Study of Influenza Outbreaks in Östergötland County, Sweden. PLoS ONE 7:e31746.

Tran CH, Sugimoto JD, Pulliam JRC, Ryan KA, Myers PD, Castleman JB, Doty R, Johnson J, Stringfellow J, Kovacevich N, Brew J, Cheung LL, Caron B, Lipori G, Harle CA, Alexander C, Yang Y, Longini IM, Jr., Halloran ME, Morris JG, Jr., and Small PA, Jr. 2014. School-Located Influenza Vaccination Reduces Community Risk for Influenza and Influenza-Like Illness Emergency Care Visits. PLOS ONE 9:e114479. 10.1371/journal.pone.0114479

van Noort SP, Águas R, Ballesteros S, Gabriela M, and Gomes M. 2012. The role of weather on the relation between influenza and influenza-like illness. Journal of Theoretical Biology 298:131-137.

Viboud C, Bjørnstad ON, Smith DL, Simonsen L, Miller MA, and Grenfell BT. 2006. Synchrony, Waves, and Spatial Hierarchies in the Spread of Influenza. Science 312:447-451.

Wallinga J, Teunis P, and Kretzschmar M. 2006. Using Data on Social Contacts to Estimate Age-specific Transmission Parameters for Respiratory-spread Infectious Agents. American Journal of Epidemiology 164:936-944.

White LF, Archer B, and Pagano M. 2014. Determining the dynamics of influenza transmission by age. Emerging themes in epidemiology 11.

World Health Organization. 2014. Influenza (Seasonal). http://www.who.int/mediacentre/factsheets/fs211/en/.

Yu H, Alonso WJ, Feng L, Tan Y, Shu Y, Yang W, and Viboud C. 2013. Characterization of Regional Influenza Seasonality Patterns in China and Implications for Vaccination Strategies: Spatio-Temporal Modeling of Surveillance Data. PLoS Medicine 10:e1001552. 


\section{FIGURES}

828 Figure 1 - Mobility flows by region made up with home-work and home-school journeys.

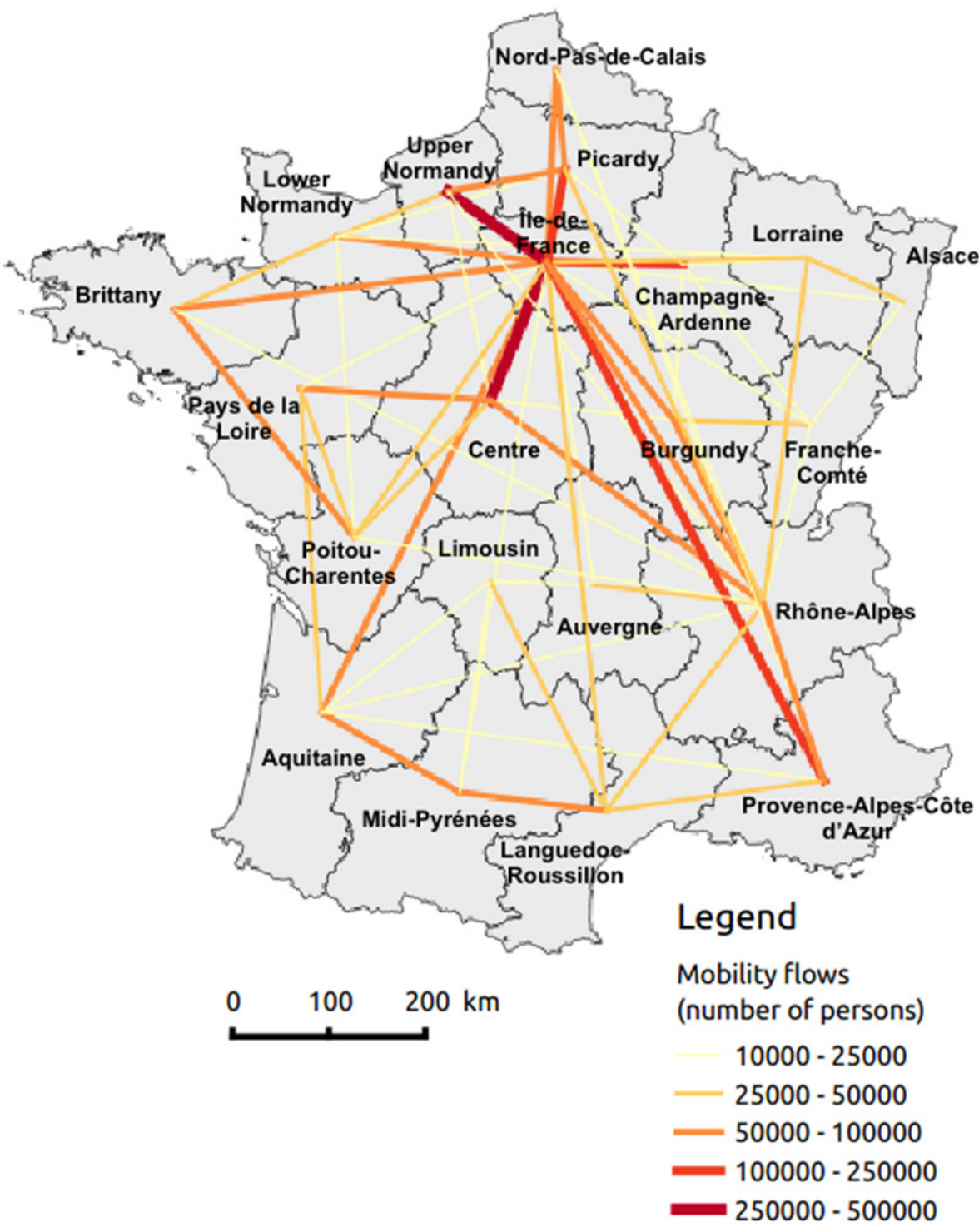

830

831 
832 Figure 2 - Variations of epidemic onset dates (scaled each year so that 0 corresponds to the first

833 week during which at least one region was in the epidemic state) between the eighteen studied

834 French regions. For all regions, we have six points (studied epidemic years), but note that some

835 of these points might be overlapping.

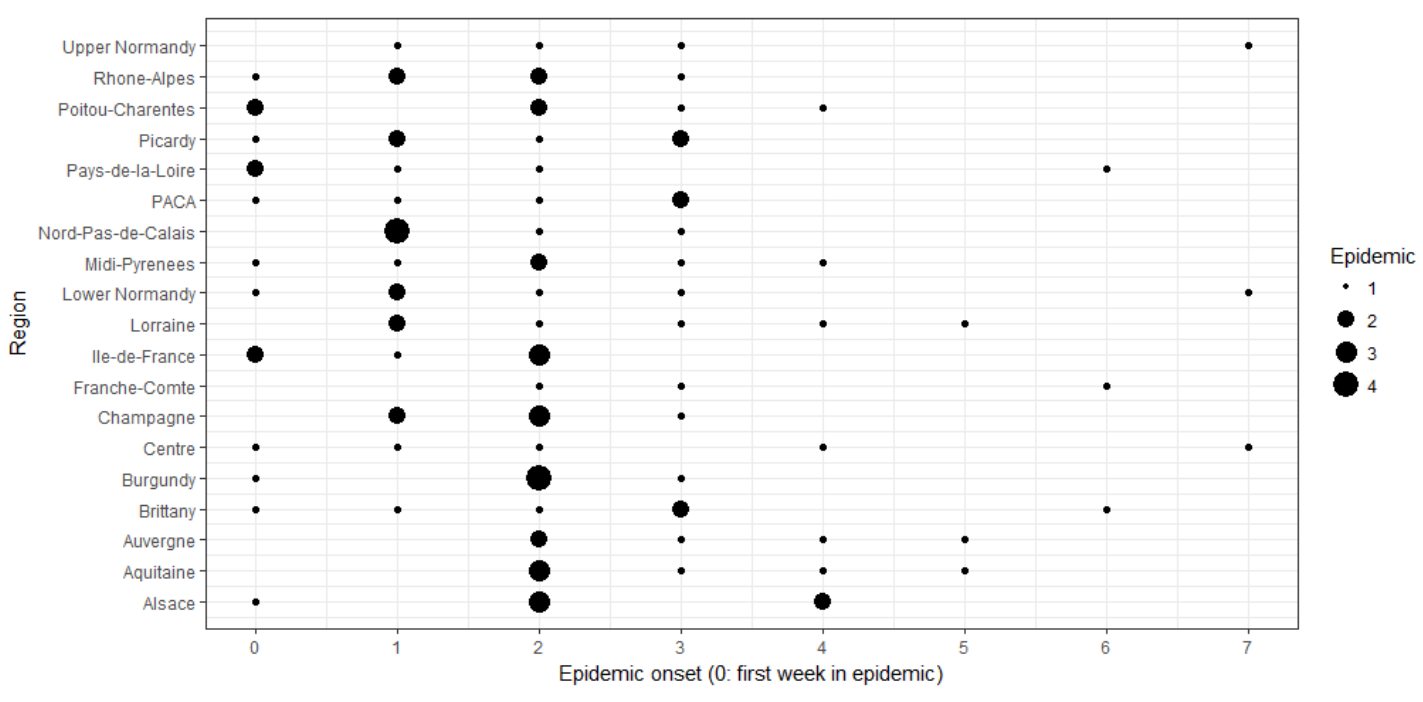

837

838 
839 Figure 3 - Epidemic onset dates of French regions according to epidemic years given by the

840 GROG network from 2006-2007 to 2012-2013 (except 2009-2010). The eighteen French regions

841 serve as replicates for the boxplots of each epidemic year.

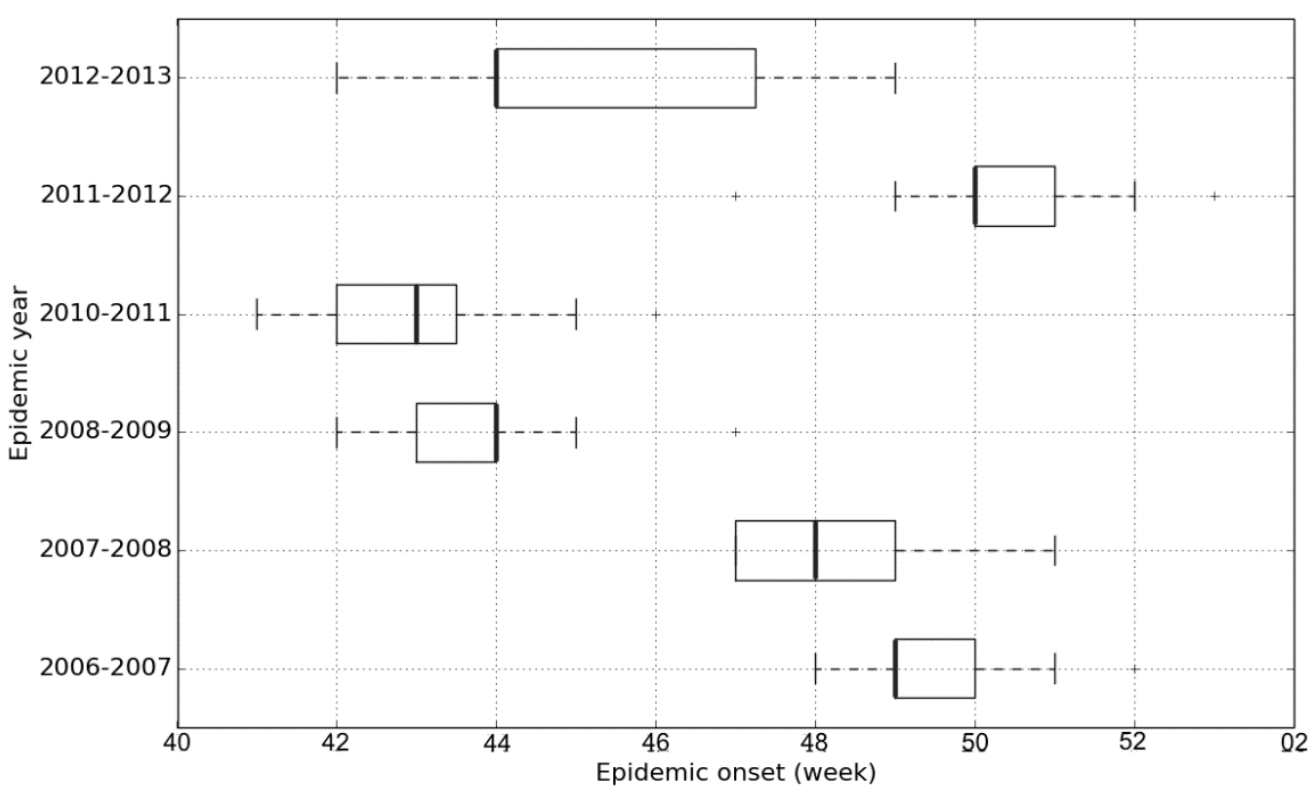

842

843

844

845

846

847 
849 Table 1 - Summary of studies about influenza timing differences

\begin{tabular}{|c|c|}
\hline Where / Scale & Data \\
\hline USA / states & $\begin{array}{l}30 \text { years, weekly influenza- } \\
\text { related mortality }\end{array}$ \\
\hline $\begin{array}{l}\text { Pennsylvania, } \\
\text { US / counties }\end{array}$ & $\begin{array}{l}6 \text { years, weekly laboratory } \\
\text { confirmed influenza cases }\end{array}$ \\
\hline $\begin{array}{l}\text { France / } \\
\text { departments }\end{array}$ & $\begin{array}{l}25 \text { years, weekly influenza } \\
\text { syndromic cases }\end{array}$ \\
\hline $\begin{array}{l}\text { France / } \\
\text { patches } 20 \mathrm{~km}\end{array}$ & $\begin{array}{l}8 \text { years, weekly influenza } \\
\text { syndromic cases }\end{array}$ \\
\hline Israel / cities & $\begin{array}{l}11 \text { years, weekly influenza } \\
\text { syndromic cases }\end{array}$ \\
\hline Brazil / states & $\begin{array}{l}22 \text { years, monthly influenza } \\
\text { related mortality }\end{array}$ \\
\hline USA / states & $\begin{array}{l}30 \text { years, weekly influenza- } \\
\text { related mortality }\end{array}$ \\
\hline $\begin{array}{l}\text { France / } \\
\text { regions }\end{array}$ & $\begin{array}{l}20 \text { years, daily influenza } \\
\text { syndromic cases }\end{array}$ \\
\hline $\begin{array}{l}\text { China / } \\
\text { provinces }\end{array}$ & $\begin{array}{l}6 \text { years, weekly laboratory } \\
\text { confirmed influenza cases }\end{array}$ \\
\hline $\begin{array}{l}\text { Canada / } \\
\text { provinces }\end{array}$ & $\begin{array}{l}11 \text { years, weekly laboratory } \\
\text { confirmed influenza cases }\end{array}$ \\
\hline USA / states & $\begin{array}{l}30 \text { years, weekly influenza- } \\
\text { related mortality }\end{array}$ \\
\hline $\begin{array}{l}\text { USA / } 271 \\
\text { cities }\end{array}$ & $\begin{array}{l}2009 \mathrm{H} 1 \mathrm{~N} 1 \text { influenza } \\
\text { pandemic weekly syndromic } \\
\text { influenza cases }\end{array}$ \\
\hline
\end{tabular}

\begin{tabular}{|c|c|}
\hline Metric & Method \\
\hline Epidemic peak & Correlation tests \\
\hline Epidemic peak & Correlation tests \\
\hline Epidemic Peak & Correlation tests \\
\hline Epidemic Peak & Correlation tests \\
\hline Epidemic Peak & Statistical test \\
\hline Epidemic Peak & Linear models \\
\hline Epidemic Peak & $\begin{array}{l}\text { Correlation tests } \\
+ \text { linear models }\end{array}$ \\
\hline Epidemic Peak & $\begin{array}{l}\text { Correlation tests } \\
+ \text { linear models }\end{array}$ \\
\hline Epidemic Peak & Linear models \\
\hline $\begin{array}{l}\text { Epidemic } 25 \% \\
\text { quantile time } \\
\text { Epidemic onset }\end{array}$ & $\begin{array}{l}\text { Generalized } \\
\text { linear model } \\
\text { Correlation test }\end{array}$ \\
\hline Epidemic onset & $\begin{array}{l}\text { Correlation tests } \\
+ \text { Mechanistic } \\
\text { models }\end{array}$ \\
\hline
\end{tabular}

Results

Correlation influenza spread / human movements (workflows) + influenza spread / population sizes

Correlation influenza spread / human movements

Correlation influenza spread / human movements (school- and work- based communing)

Correlation number of influenza cases / density

\section{Highly synchronized epidemics}

Spatial correlation suggesting a role of climate (temperature and humidity)

Correlation influenza spread / air-traffic

Correlation influenza spread/train- and automobiletraffic

Strong correlation influenza spread / climatic factors (temperature, sunshine, rainfall), weaker correlation influenza spread / human movements

Correlation influenza spread / temperature, absolute humidity, population size and spatial ordering

Correlation epidemic onsets / absolute humidity

Strong correlation influenza onsets/school opening + short spatial diffusion, weaker correlation influenza onset / population sizes, absolute humidity
Reference

(Viboud et 2006)

(Stark et al. 2012)

(Charaudea al. 2014)

(Bonabeau 1998)

(Barnea et a 2014; Hupp et al. 2012)

(Alonso et a 2007)

(Crépey \& Barthélemy 2007)

(Crépey \& Barthélemy 2007)

(Yu et al. 2

(He et al. 2(

(Shaman et 2010)

(Gog et al.

2014) 
851 Table 2 - Preliminary analysis: evaluating the relevant scales of variation of the different

852 variables (considered each separately) using the (preliminary) linear mixed model. The

853 importance of variations at the different scales is quantified by the corresponding estimated

854 standard deviations (residuals and from random - regions, years and weeks - effects).

$\begin{array}{llllll}\text { Factors } & \begin{array}{l}\text { Intercept } \\ \text { (average) }\end{array} & \begin{array}{l}\text { Regions } \\ \text { (standard }\end{array} & \begin{array}{c}\text { Years } \\ \text { (standard }\end{array} & \begin{array}{c}\text { Weeks } \\ \text { (standard }\end{array} & \begin{array}{c}\text { Residuals } \\ \text { (standard }\end{array}\end{array}$
deviation, $\widehat{\sigma_{R}}$ ) deviation, $\left.\widehat{\sigma_{Y}}\right) \quad$ deviation, $\left.\widehat{\sigma_{W}}\right)$ deviation, $\left.\hat{\sigma}\right)$

\begin{tabular}{cccccc}
\hline \hline Epidemic onset (week) & 6.95 & 1.50 & 1.69 & - & 3.83 \\
Population size (inhabitant) & $3,100,600$ & $2,481,281$ & 34,209 & - & 4,1887 \\
Proportion of children & 0.24 & 0.014 & 0.002 & - & 0.001 \\
Temperature $\left({ }^{\circ} \mathrm{C}\right)$ & 6.70 & 0.86 & 1.18 & 2.78 & 2.69 \\
Absolute humidity $\left(\mathrm{g} / \mathrm{m}^{3}\right)$ & 6.43 & 0.37 & 0.54 & 1.12 & 1.08 \\
855 & & & &
\end{tabular}

855

856

857

858 
859 Table 3 - Summary of the studied covariates (whose link with epidemic onset dates was tested)

860 with associated sub-covariates, model parameters, scales of variation and indexes permuted.

\begin{tabular}{ccccc} 
Covariate & Sub-covariate & $\begin{array}{c}\text { Associated } \\
\text { parameter }\end{array}$ & Scale & $\begin{array}{c}\text { Permuted } \\
\text { index }\end{array}$ \\
\hline \hline \multirow{2}{*}{ Temperature } & $T W_{W}$ & $a_{T W}$ & Global & Weeks \\
& $T Y_{Y, W}$ & $a_{T R}$ & Spatial & Regions \\
& $T r s_{R, Y, W}$ & $a_{T Y}$ & Annual & Years \\
& $H W_{W}$ & $a_{T r e s}$ & Spatiotemporal & Regions and \\
& $H R_{R}$ & & & years \\
\hline Absolute & $H Y_{Y, W}$ & $a_{H W}$ & Global & Weeks \\
Humidity & $a_{H R}$ & Spatial & Regions \\
& $H r S_{R, Y, W}$ & $a_{H Y}$ & Annual & Years \\
& $a_{H r e s}$ & Spatiotemporal & Regions and \\
& & & & years
\end{tabular}

\begin{tabular}{ccccc}
\hline Mobility $\quad \sum_{i=1, i \neq r}^{N}\left(\delta_{r i}+\delta_{i r}\right) \times \frac{I_{i}(t)}{S_{i}}$ & - & Spatiotemporal & Regions
\end{tabular}

\begin{tabular}{cllll}
\hline $\begin{array}{c}\text { Population } \\
\text { size }\end{array}$ & $S_{R}$ & $a_{S}$ & Spatial & Regions \\
\hline $\begin{array}{c}\text { Proportion of } \\
\text { children }\end{array}$ & $C_{R}$ & $a_{C}$ & Spatial & Regions \\
& & & &
\end{tabular}

861

862

863 
864 Table 4 - Estimates of the associated parameter tested for each covariate with the $p$ value of the 865 associated permutation test. For each covariate, all these pieces of information come from the 866 model used to evaluate the link between the covariate and epidemic onset dates.

\begin{tabular}{cccc} 
Covariate & Symbol & Estimate & P value \\
\hline \hline T: global & $T W_{W}$ & -0.4932 & 0.1718 \\
T: spatial & $T R_{R}$ & -0.2557 & 0.1598 \\
T: annual & $T Y_{Y, W}$ & -0.3841 & 0.2627 \\
T: spatiotemporal & $T r e s_{R, Y, W}$ & 0.0461 & 0.9361 \\
H: global & $H W_{W}$ & -0.0200 & 0.1089 \\
H: spatial & $H R_{R}$ & -0.4763 & 0.0290 \\
H: annual & $H Y_{Y, W}$ & -0.0449 & 0.7512 \\
H: spatiotemporal & $H r e S_{R, Y, W}$ & -0.3004 & 0.7932 \\
Mobility flows: corrected & $\sum_{i=1, i \neq r}^{N}\left(\delta_{r i}+\delta_{i r}\right) \times \frac{I_{i}(t)}{S_{i}}$ & - & 0.5704 \\
Mobility flows: uncorrected & $\sum_{i=1, i \neq r}^{N}\left(\delta_{r i}+\delta_{i r}\right) \times \frac{I_{i}(t)}{S_{i}}$ & - & 0.7333 \\
Population size & $\log \left(S_{R}\right)$ & 0.1274 & 0.1718 \\
Proportion of children & $C_{R}$ & 0.1215 & 0.0929
\end{tabular}

867

868

869

870 